\title{
Direct dark matter searches via pseudo-scalar portal
}

\author{
Paolo Panci \\ Institut d'Astrophysique, CNRS, UMR $7095 \&$ Université Pierre et Marie Curie, \\ 98bis Boulevard Arago, F-75014 Paris, France \\ E-mail: panci@iap.fr
}

\begin{abstract}
We study a Dirac Dark Matter (DM) particle interacting with ordinary matter via a pseudo-scalar portal, and analyze its impact on direct detection experiments. This candidate can accommodate the long-standing DAMA modulated signal and yet be compatible with all exclusion limits at $99_{S} \% \mathrm{CL}$. This result holds for natural choices of the pseudo-scalar-quark couplings, and give rise to a significant enhancement of the DMproton coupling with respect to the coupling to neutrons. The model could be tested with measurements of rare meson decays, flavor changing processes, and searches for axion-like particles with mass in the $\mathrm{MeV}$ range.
\end{abstract}

Keywords: MG14 Proceedings.

\section{Introduction}

Direct searches for DM aim at detecting the tiny nuclear recoils arising from scattering between DM particles and target nuclei in underground detectors. Direct detection experiments have underwent astonishing developments in recent years, achieving unprecedented sensitivity to Weakly Interacting Massive Particles (WIMPs) in the mass range from few $\mathrm{GeV}$ to tens of $\mathrm{TeV}$. The most stringent limits on the DM parameter space are set by LUX ${ }^{1}$, XENON100 $^{2}$, and SuperCDMS ${ }^{3}$ for SpinIndependent (SI) interactions, with $\mathrm{PICASSO}^{4}, \mathrm{SIMPLE}^{5}, \mathrm{COUPP}^{6}$, and $\mathrm{KIMS}^{7}$ setting relevant bounds for Spin-Dependent (SD) interactions and DM-proton couplings. While these and other searches did not find evidences for DM, four experiments have signals that can be interpreted as due to WIMP scatterings ${ }^{8-11}$. The significance of the excesses is mild (from $2 \sigma$ to $4 \sigma$ ), except for DAMA's result ${ }^{12}$, where the observation of an annually modulated rate as expected from the simplest model of DM halo, reaches the very high significance of $9.3 \sigma$. This achievement however has received a long-standing series of criticisms, given that the interpretation of the DAMA data in the light of many models of WIMP interactions is incompatible with all exclusion bounds.

The scope of this work is to explore how different NR DM-nucleus interactions, can alter the allowed regions of the positive results experiments and the constraints coming from null results. In particular in Sec. 2, I quickly review a new and more general formalism to study signals in direct searches based on nonrelativistic operators. In Sec. 3 I show that a DM particle interacting with ordinary matter via the exchange of a light pseudo-scalar, can accommodate the DAMA data while being compatible with all null direct DM searches. Finally in Sec. 3 I conclude with a discussion on the complementary constraints coming from rare meson decays and fixed target experiments. 


\section{Basics and formalism of non-relativistic operators}

In direct searches the main quantity that one has to compute is the differential rate of nuclear recoil measured in $\mathrm{cpd} / \mathrm{kg} / \mathrm{keV}$. For a target nuclide $\mathcal{N}$ at rest, recoiling with energy $E_{\mathrm{R}}$ with a DM particle with initial velocity $v$ and mass $m_{\mathrm{DM}}$, it reads

$$
\frac{\mathrm{d} R_{\mathcal{N}}}{\mathrm{d} E_{\mathrm{R}}}=\frac{\xi_{\mathcal{N}}}{m_{\mathcal{N}}} \frac{\rho_{\odot}}{m_{\mathrm{DM}}} \int_{v_{\min }\left(E_{\mathrm{R}}\right)}^{v_{\mathrm{esc}}} \mathrm{d}^{3} v v f_{\mathrm{E}}(\vec{v}) \frac{\mathrm{d} \sigma_{\mathcal{N}}}{\mathrm{d} E_{\mathrm{R}}}\left(v, E_{\mathrm{R}}\right),
$$

where $\rho_{\odot}$ is the local DM energy density, $m_{\mathcal{N}}$ is the mass of the target nuclide and $\xi_{\mathcal{N}}$ is its mass fraction in the detector. Here the differential cross section $\mathrm{d} \sigma_{\mathcal{N}} / \mathrm{d} E_{\mathrm{R}}$ is weighted with the DM velocity distribution in the Earth's frame $f_{\mathrm{E}}(\vec{v})$ which is modulated in time due to the Earth's motion around the Sun during the years ${ }^{13}$. In the velocity integral above, $v_{\min }\left(E_{\mathrm{R}}\right)$ is the minimal velocity providing a nuclear recoil $E_{\mathrm{R}}$ of the nuclide and $v_{\text {esc }}$ is the Milky Way's escape velocity. For elastic collision, $v_{\min }\left(E_{\mathrm{R}}\right)=\sqrt{m_{\mathcal{N}} E_{\mathrm{R}} /\left(2 \mu_{\mathcal{N}}^{2}\right)}$, where $\mu_{\mathcal{N}}=m_{\mathrm{DM}} m_{\mathcal{N}} /\left(m_{\mathrm{DM}}+m_{\mathcal{N}}\right)$ is the DM-nucleus reduced mass.

In general, the differential cross section in the non-relativistic regime, writes

$$
\frac{\mathrm{d} \sigma_{\mathcal{N}}}{\mathrm{d} E_{\mathrm{R}}}\left(v, E_{\mathrm{R}}\right)=\frac{1}{32 \pi} \frac{1}{m_{\mathrm{DM}}^{2} m_{\mathcal{N}}} \frac{1}{v^{2}} \overline{\left|\mathcal{M}_{\mathcal{N}}\right|^{2}},
$$

where $\overline{\left|\mathcal{M}_{\mathcal{N}}\right|^{2}}$ is the square of the DM-nucleus matrix element that contains all the information related to the nature of the interactions and the nuclear physics.

We know that the local DM velocity is much smaller than the speed of light, therefore the right formalism that let better describes the physics of the scattering is the one of Non-Relativistic (NR) operators. In fact, since for elastic collisions, the relevant degrees of freedom are the exchanged momentum $\vec{q}$, the DM-nucleon relative velocity $\vec{v}$, the nucleon $\operatorname{spin} \vec{s}_{N}(N=p, n$ can be proton or neutron $)$ and eventually the DM one $\vec{s}_{\chi}$ if different from zero, the scattering amplitude at the level of the nucleons will be a rotationally invariant function of these variables. In this regards, a basis of 16 Galilean invariant operators $\left(\mathcal{O}_{i}^{\mathrm{NR}}\right)$ can be constructed and therefore the DM-nucleon matrix element $\mathcal{M}_{N}$ can be expressed as a linear combination of them. In particular $\mathcal{M}_{N}=\sum_{i=1}^{16} \mathfrak{c}_{i}^{N}\left(\lambda, m_{\mathrm{DM}}\right) \mathcal{O}_{i}^{\mathrm{NR}}$, where the coefficients $\mathfrak{c}_{i}^{N}\left(\lambda, m_{\mathrm{DM}}\right)$ are functions of the free parameters of the underlying relativistic theory (collectively denoted by $\lambda$ ), and the mass of the DM particle $m_{\mathrm{DM}}$. An almost complete list of these Galilean invariant combinations is given by

$$
\begin{array}{lll}
\mathcal{O}_{1}^{\mathrm{NR}}=\mathbb{1}, & \mathcal{O}_{2}^{\mathrm{NR}}=\left(v^{\perp}\right)^{2}, \\
\mathcal{O}_{3}^{\mathrm{NR}}=i \vec{s}_{N} \cdot\left(\vec{q} \times \vec{v}^{\perp}\right), & \mathcal{O}_{4}^{\mathrm{NR}}=\vec{s}_{\chi} \cdot \vec{s}_{N}, \\
\mathcal{O}_{5}^{\mathrm{NR}}=i \vec{s}_{\chi} \cdot\left(\vec{q} \times \vec{v}^{\perp}\right), & \mathcal{O}_{6}^{\mathrm{NR}}=\left(\vec{s}_{\chi} \cdot \vec{q}\right)\left(\vec{s}_{N} \cdot \vec{q}\right), \\
\mathcal{O}_{7}^{\mathrm{NR}}=\vec{s}_{N} \cdot \vec{v}^{\perp}, & & \mathcal{O}_{8}^{\mathrm{NR}}=\vec{s}_{\chi} \cdot \vec{v}^{\perp}, \\
\mathcal{O}_{9}^{\mathrm{NR}}=i \vec{s}_{\chi} \cdot\left(\vec{s}_{N} \times \vec{q}\right), & \mathcal{O}_{10}^{\mathrm{NR}}=i \vec{s}_{N} \cdot \vec{q}, \\
\mathcal{O}_{11}^{\mathrm{NR}}=i \vec{s}_{\chi} \cdot \vec{q}, & & \mathcal{O}_{12}^{\mathrm{NR}}=\vec{v}^{\perp} \cdot\left(\vec{s}_{\chi} \times \vec{s}_{N}\right), \ldots \ldots,
\end{array}
$$


where we follow the numbering adopted in Refs. ${ }^{14-16}$. There are other 4 operators one can write. Neverthless, since they are suppressed by higher power of $\vec{v}^{\perp}$ or $\vec{q}$, we omit them in the list above.

Since now the nucleus is made of neutrons and protons, one has to correct the DM-nucleon matrix element with the nuclear responses that take into account the finite size of the target. According to Eq. (55) of ${ }^{14}$ we can write the spin-averaged amplitude squared for scattering off a target nucleus as

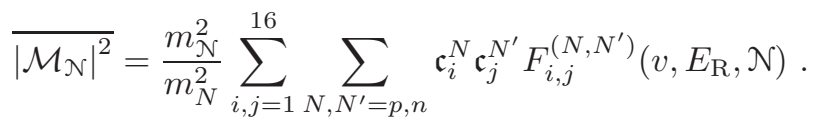

The functions $F_{i, j}^{\left(N, N^{\prime}\right)}\left(v, E_{\mathrm{R}}, \mathcal{N}\right)$ are the nuclear responses and they encode all the information coming from the NR nuclear physics. A complete set of them for each pair of operators $(i, j)$, pair of nucleons $\left(N, N^{\prime}\right)$, and for several target nuclei $\mathcal{N}$, has been for the first time provided in the appendices of Ref. ${ }^{14}$. This is extremely useful because in this way all the possible NR DM-nucleus interactions can be studied.

Plugging back Eq. (4) in Eq. (2), the differential rate of nuclear recoil can be cast in a very general way. Following Refs. ${ }^{16,17}$, it writes

$$
\frac{\mathrm{d} R_{\mathcal{N}}}{\mathrm{d} E_{\mathrm{R}}}=X \xi_{\mathcal{N}} \sum_{i, j=1}^{16} \sum_{N, N^{\prime}=p, n} \mathfrak{c}_{i}^{N}\left(\lambda, m_{\mathrm{DM}}\right) \mathfrak{c}_{j}^{N^{\prime}}\left(\lambda, m_{\mathrm{DM}}\right) \mathcal{F}_{i, j}^{\left(N, N^{\prime}\right)}\left(E_{\mathrm{R}}, \mathcal{N}\right)
$$

where the constant $X \equiv \rho_{\odot} /(32 \pi) \cdot 1 /\left(m_{\mathrm{DM}}^{3} m_{N}^{2}\right)$ and

$$
\mathcal{F}_{i, j}^{\left(N, N^{\prime}\right)}\left(E_{\mathrm{R}}, \mathcal{N}\right) \equiv \int_{v_{\min }\left(E_{\mathrm{R}}\right)}^{v_{\mathrm{esc}}} \mathrm{d}^{3} v \frac{1}{v} f_{\mathrm{G}}\left(\vec{v}+\vec{v}_{\mathrm{E}}(t)\right) F_{i, j}^{\left(N, N^{\prime}\right)}\left(v, E_{\mathrm{R}}, \mathcal{N}\right) .
$$

To properly reproduce now the measured recoil rate and in turn the expected number of events in a given experiment, we need to take into account the characteristics of the detector. In so doing, we have to convolve Eq. (15) with the resolution of the detector $\mathcal{K}_{\mathcal{N}}\left(E_{\mathrm{R}}, E^{\prime}\right)$ and the efficiency function $\epsilon\left(E^{\prime}\right)$. Collected the elements commented upon above, we can finally write the expected number of events in the $k^{\text {th }}$-esime energy bin of the detector as

$$
N_{k}^{\mathrm{th}}=X \sum_{i, j=1}^{16} \sum_{N, N^{\prime}=p, n} \mathfrak{c}_{i}^{N}\left(\lambda, m_{\mathrm{DM}}\right) \mathfrak{c}_{j}^{N^{\prime}}\left(\lambda, m_{\mathrm{DM}}\right) \tilde{\mathcal{F}}_{i, j}^{\left(N, N^{\prime}\right)}\left(m_{\mathrm{DM}}, k\right) .
$$

where the functions

$$
\tilde{\mathcal{F}}_{i, j}^{\left(N, N^{\prime}\right)}\left(m_{\chi}, k\right)=w_{k} \sum_{T} \xi_{T} \int_{\Delta E_{k}} \mathrm{~d} E^{\prime} \epsilon\left(E^{\prime}\right) \int_{0}^{\infty} \mathrm{d} E_{\mathrm{R}} \mathcal{K}_{\mathcal{N}}\left(E_{\mathrm{R}}, E^{\prime}\right) \mathcal{F}_{i, j}^{\left(N, N^{\prime}\right)}\left(E_{\mathrm{R}}, \mathcal{N}\right),
$$

are a sort of integrated form factors that encodes all the information related to astrophysics (in the velocity distribution), nuclear physics (in the nuclear responses) and the detector dependency of the rate. Here $w_{k}$ is the exposure (expressed in $\mathrm{kg}$ per days) and $\Delta E_{k}$ is the width of the $k^{\text {th }}$-esime energy bin. There is just one 
of these factors for each energy bin $k$ of a given experiment, and for each pair of operators $(i, j)$ and pair of nucleons $\left(N, N^{\prime}\right)$. Therefore, once one has computed all of these finite number of integrated form factors the expected number of events can be obtained for any kind of interactions whose particle physics is completely encapsulated in the coefficient $\mathfrak{c}_{i}^{N}\left(\lambda, m_{\mathrm{DM}}\right)$.

In this way, the model dependent results presented by the experimental collaboration in terms of the "standard" SI and SD cross section can also be applied for other class of models characterized by a different DM-nucleus interaction. Several authors (see e.g. Refs. ${ }^{15-19}$ ) have used this new formalism in order to explore how different DM-nucleus interactions, described in the NR limit by different operators and in turn integrated form factors, can alter the allowed regions of the positive results experiments and the constraints coming from null results. In particular, in the next section we show that a DM particle interacting with ordinary matter via the exchange of a light pseudo-scalar (this model in the NR limit is described by the operator $\left.\mathcal{O}_{6}^{\mathrm{NR}}=\left(\vec{s}_{\chi} \cdot \vec{q}\right)\left(\vec{s}_{N} \cdot \vec{q}\right)\right)$, can accommodate the DAMA data while being compatible with all null direct DM searches.

Before moving on with the details of the DM model, it is worth classifying here all the possible NR operators in two main classes. The operators which actually are independent on the spin of the nucleon:

$$
\begin{array}{ll}
\mathcal{O}_{1}^{\mathrm{NR}}=\mathbb{1}, & \mathcal{O}_{2}^{\mathrm{NR}}=\left(v^{\perp}\right)^{2}, \\
\mathcal{O}_{5}^{\mathrm{NR}}=i \vec{s}_{\chi} \cdot\left(\vec{q} \times \vec{v}^{\perp}\right), & \mathcal{O}_{8}^{\mathrm{NR}}=\vec{s}_{\chi} \cdot \vec{v}^{\perp}, \\
\mathcal{O}_{11}^{\mathrm{NR}}=i \vec{s}_{\chi} \cdot \vec{q}, &
\end{array}
$$

and the ones which instead depend on the nucleon spin:

$$
\begin{array}{ll}
\mathcal{O}_{3}^{\mathrm{NR}}=i \vec{s}_{N} \cdot\left(\vec{q} \times \vec{v}^{\perp}\right), & \mathcal{O}_{4}^{\mathrm{NR}}=\vec{s}_{\chi} \cdot \vec{s}_{N}, \\
\mathcal{O}_{6}^{\mathrm{NR}}=\left(\vec{s}_{\chi} \cdot \vec{q}\right)\left(\vec{s}_{N} \cdot \vec{q}\right), & \mathcal{O}_{7}^{\mathrm{NR}}=\vec{s}_{N} \cdot \vec{v}^{\perp}, \\
\mathcal{O}_{9}^{\mathrm{NR}}=i \vec{s}_{\chi} \cdot\left(\vec{s}_{N} \times \vec{q}\right), & \mathcal{O}_{10}^{\mathrm{NR}}=i \vec{s}_{N} \cdot \vec{q}, \\
\mathcal{O}_{12}^{\mathrm{NR}}=\vec{v}^{\perp} \cdot\left(\vec{s}_{\chi} \times \vec{s}_{N}\right) . &
\end{array}
$$

This classification is needed, because direct detection experiments based on the double-phase xenon technology (e.g. LUX or XENON) are particularly sensitive to the SI operators in Eq. (9). Hence, even with different velocity and momenta dependences in the differential cross sections with respect to the standard SI interaction $\left(\mathcal{O}_{1}^{N R}\right)$, will be extremely difficult or impossible to reconcile the DAMA results with the constraints coming from null results experiments.

On the other hand, if one has a spin dependent interaction, the situation here is more interesting because an experiment like DAMA, which employs both the sodium and iodine with unpaired protons in the outer nuclear shells, is sensitive to the DMproton SD interaction, while and experiment like LUX, which employs the xenon with unpaired neutrons is blind to such interaction. Therefore, the complicated experimental puzzle the experiments in direct searches has left to us, can probably solved if one has a relativistic DM model that in the NR limit ends up with one 
of the SD NR operators in Eq. (10), in which the DM coupling with the protons is much larger than that with the neutrons.

In the next section, we show that exist at least one relativistic DM model that in the NR limit ends up with a longitudinal SD interaction described by the operator $\mathcal{O}_{6}^{\mathrm{NR}}$ in which, the DM coupling with the protons is naturally much larger than the one with the neutrons.

\section{The Dark Matter model}

The DM is a Dirac fermion $\chi$, which interacts, with a coupling $g_{\mathrm{DM}}$, with a (real) pseudo-scalar $a$ with mass $m_{a}$ coupled to the SM fermions:

$$
\mathscr{L}_{\mathrm{int}}=-i \frac{g_{\mathrm{DM}}}{\sqrt{2}} a \bar{\chi} \gamma_{5} \chi-i g \sum_{f} \frac{g_{f}}{\sqrt{2}} a \bar{f} \gamma_{5} f .
$$

As follows we will consider two types of fermion couplings $g_{f}$ : flavor-universal couplings $g_{f}=1$ independent of the fermion type, and Higgs-like couplings proportional to the fermion masses $g_{f}=m_{f} / 174 \mathrm{GeV}$. Furthermore, for the direct detection analysis we will consider also the case of DM coupled equally to protons and neutrons (isoscalar interaction, ${ }^{\mathrm{a}}$ also called "isospin-conserving"), as assumed e.g. by ${ }^{18,19}$. In all cases we denote with $g$ a multiplicative factor common to all couplings of $a$ with SM fermions.

When computing scattering cross sections at direct detection experiments, it is necessary to bear in mind that the scattering occurs with the whole nucleus due to the small WIMP speed. Therefore, starting with an interaction Lagrangian with quarks as in Eq. (11), one needs first to determine the DM-nucleon effective Lagrangian and then to properly take into account the composite structure of the nucleus which results in the appearance of nuclear form factors in the cross section.

The first step is accomplished in our case by taking the following effective DMnucleon interaction Lagrangian, valid in the regime of contact-interaction:

$$
\mathscr{L}_{\text {eff }}=\frac{1}{2 \Lambda_{a}^{2}} \sum_{N=p, n} g_{N} \bar{\chi} \gamma^{5} \chi \bar{N} \gamma^{5} N
$$

where $\Lambda_{a} \equiv m_{a} / \sqrt{g_{\mathrm{DM}} g}$. The proton and neutron coupling constants are given by

$$
g_{N}=\sum_{q=u, d, s} \frac{m_{N}}{m_{q}}\left[g_{q}-\sum_{q^{\prime}=u, \ldots, t} g_{q^{\prime}} \frac{\bar{m}}{m_{q^{\prime}}}\right] \Delta_{q}^{(N)}
$$

\footnotetext{
a Notice that our use of the term 'isoscalar' refers to the isospin symmetry between proton and neutron. As it will become clear later on this does not imply, nor is implied by, isospin symmetry at the quark level.
} 
where $\bar{m} \equiv\left(1 / m_{u}+1 / m_{d}+1 / m_{s}\right)^{-1}$ and we use

$$
\begin{aligned}
& \Delta_{u}^{(p)}=\Delta_{d}^{(n)}=+0.84, \\
& \Delta_{d}^{(p)}=\Delta_{u}^{(n)}=-0.44, \\
& \Delta_{s}^{(p)}=\Delta_{s}^{(n)}=-0.03
\end{aligned}
$$

for the quark spin content of the nucleon ${ }^{20}$.

It is important to notice here that $g_{p}$ is naturally larger (in modulus) than $g_{n}$ in both the flavor-universal and Higgs-like coupling scenarios. This, as mentioned at the end of Sec. 2, will have important phenomenological consequences. In fact, since the interaction in Eq. (12) measures a certain component of the spin content of the nucleus carried by nucleons ${ }^{14}$, a large $g_{p} / g_{n}$ will favor those nuclides (like ${ }^{23} \mathrm{Na},{ }^{127} \mathrm{I}$ and ${ }^{19} \mathrm{~F}$ ) with a large spin due to their unpaired proton rather than ${ }^{129,131}$ Xe nuclei with an unpaired neutron. Given that the most stringent bounds for most DM-nucleus interactions are given at present by experiments using xenon $(\text { LUX, XENON100) })^{\mathrm{b}}$ while DAMA employs sodium and iodine, a large value of $g_{p} / g_{n}$ would go in the direction of reconciling them. From the values in Eq. (14) we get $g_{p} / g_{n}=-16.4$ for flavor-universal and -4.1 for Higgs-like interactions. The relative size of the two couplings depends on the actual values of the $\Delta_{q}^{(N)}$ 's, which are uncertain (see e.g. Table 4 in $^{16}$ for a comparison of the different values found in the literature); the values in Eq. (14) are conservative in the sense that they minimize the ratio $g_{p} / g_{n}$, respect to what obtained with other choices of the $\Delta_{q}^{(N)}$ 's. Finally we will also use isoscalar interactions, i.e. by setting $g=g_{p}=g_{n}$ without using Eq. (13), as assumed in ${ }^{18,19}$.

Once the DM-nucleon Lagrangian is established, one needs to determine the DM interaction cross section with the nucleus. This is customarily done by coherently adding the amplitudes of interaction with the different nucleons in the nucleus, and multiplying by an appropriate nuclear form factor that parametrizes the loss of coherence in the scattering with increasing exchanged momentum (see Eqs. (2) and (4)). While form factors for the standard SI and SD interactions have been extensively studied, little is known of form factors for other interactions. Notice that the Lagrangian in Eq. (12) corresponds in the NR limit to a DM-nucleon interaction $\mathcal{O}_{6}^{\mathrm{NR}}=\left(\vec{s}_{\chi} \cdot \vec{q}\right)\left(\vec{s}_{N} \cdot \vec{q}\right)$, while the standard spin-dependent interaction corresponds to $\mathcal{O}_{4}^{\mathrm{NR}}=\vec{s}_{\chi} \cdot \vec{s}_{N}$. At the nuclear level, the difference stands in the fact that the former interaction only measures the component of the nucleon spin in the nucleus that is longitudinal to $\vec{q}$, while the latter couples to both longitudinal and transverse components. Therefore it is not justified to use the standard SD form factor for the interaction in Eq. (12) as done e.g. in ${ }^{21,22}$, although in some cases it could be used as a proxy ${ }^{19}$. The form factor to be used in this case has been computed, as mentioned in Sec. 2 , in Ref. ${ }^{14}$ using standard shell model techniques.

\footnotetext{
${ }^{\mathrm{b}}$ We do not consider germanium detectors as their sensitivity to SD interaction via unpaired protons is smaller than e.g. COUPP in the mass range relevant for our model.
} 

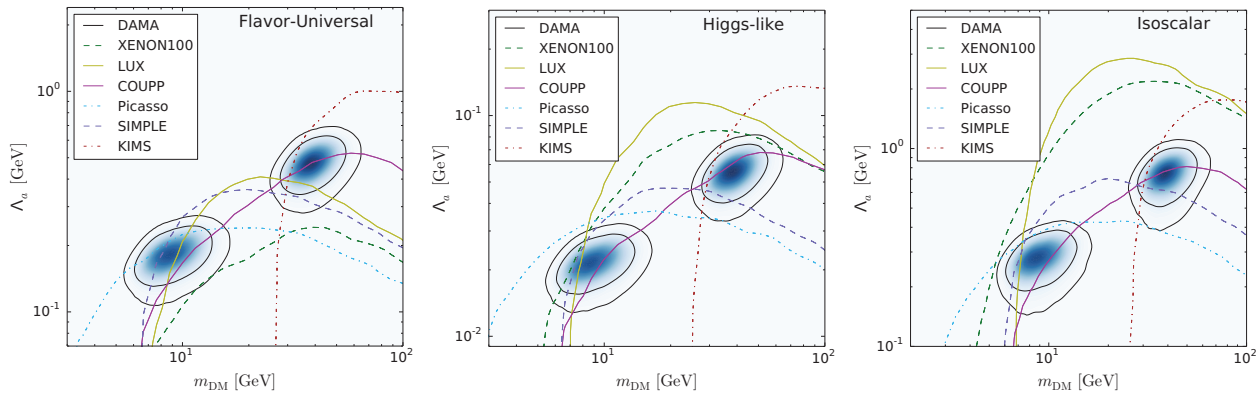

Fig. 1. 2-dimensional credible regions for DAMA (shaded/black solid, $90 \%$ and $99 \%$ CL) and exclusion limits $\left(99_{S} \% \mathrm{CL}\right)$ in the $\left(m_{\mathrm{DM}}, \Lambda_{a}\right)$ plane, for flavor-universal (left), Higgs-like (center) and isoscalar (right) couplings. This figure is taken from Ref. ${ }^{23}$.

For our model, the differential rate of nuclear recoils writes

$$
\frac{\mathrm{d} R_{\mathcal{N}}}{\mathrm{d} E_{\mathrm{R}}}=X \xi_{\mathcal{N}} \sum_{N, N^{\prime}=p, n} \mathfrak{c}_{6}^{N}\left(\Lambda_{a}, m_{\mathrm{DM}}\right) \mathfrak{c}_{6}^{N^{\prime}}\left(\Lambda_{a}, m_{\mathrm{DM}}\right) \mathcal{F}_{6,6}^{\left(N, N^{\prime}\right)}\left(E_{\mathrm{R}}, \mathcal{N}\right)
$$

where $\mathfrak{c}_{6}^{N}\left(\Lambda_{a}, m_{\mathrm{DM}}\right)=2 g_{N} / \Lambda_{a}^{2}$ can be obtained by performing the NR limit of the Lagrangian in Eq. $(12)^{16}$ and $\mathcal{F}_{6,6}^{\left(N, N^{\prime}\right)}\left(E_{\mathrm{R}}, \mathcal{N}\right)=q^{4} / 16 F_{\Sigma^{\prime \prime}}^{\left(N, N^{\prime}\right)}\left(q^{2}\right)$ is given in the appendix of Ref. ${ }^{14}$. The large suppression factor $q^{4} / \Lambda_{a}^{4}=g_{\mathrm{DM}}^{2} g^{2} \cdot q^{4} / m_{a}^{4}$ for large $m_{a}$ is the reason why the interaction in Eq. (12) has often been neglected.

In Ref. ${ }^{23}$, we have analyzed data by LUX, XENON100, PICASSO, SIMPLE, COUPP, KIMS and DAMA. We have used Bayesian statistics to infer the $99_{S} \%$ credible interval for the exclusion limits and both the $90 \%$ and $99 \%$ credible regions for DAMA from the posterior probability density function. We have considered log priors for both our relevant parameters: the DM mass $m_{\mathrm{DM}}$, from $1 \mathrm{GeV}$ to $1 \mathrm{TeV}$, and the scale $\Lambda_{a}$, from $0.01 \mathrm{GeV}$ to $100 \mathrm{GeV}$, not to favor a particular mass scale range. For each experiment we have marginalized over the nuisance parameters, given by the uncertain astrophysical parameters $\rho, v_{0}, v_{\text {esc }}$ (the central values for the Gaussian priors are $\bar{\rho}=0.3 \mathrm{GeV} / \mathrm{cm}^{3}, \bar{v}_{0}=230 \mathrm{~km} / \mathrm{s}$ and $\bar{v}_{\text {esc }}=544 \mathrm{~km} / \mathrm{s}$ ), as well as the experimental uncertainties.

Fig. 1, taken again from Ref. ${ }^{23}$, shows the results for three choices of couplings: flavor-universal, Higgs-like and isoscalar. The two DAMA regions correspond respectively to scattering off $\mathrm{Na}$ (peaked around $m_{\mathrm{DM}} \sim 8 \mathrm{GeV}$ ) and I (peaked around $\left.m_{\mathrm{DM}} \sim 40 \mathrm{GeV}\right)$. Part of the regions is compatible with all null experiments for flavor-universal couplings at $99_{S} \% \mathrm{CL}$. It is worth noticing how the large enhancement of the WIMP-proton coupling with respect to the WIMP-neutron coupling suppresses the LUX and XENON100 bounds but not COUPP, PICASSO, SIMPLE and KIMS. For Higgs-like couplings the LUX and XENON100 bounds are less suppressed due to the reduced $g_{p} / g_{n}$ enhancement, and the exclusion limits disfavor both sodium and iodine regions. In the isoscalar case instead there is no enhancement and DAMA is largely disfavored at $99_{S} \%$ CL by both XENON100 and LUX. 


\section{Conclusions}

In this work, we have shown that a Dirac DM particle interacting with ordinary matter via the exchange of a light pseudo-scalar can accommodate the DAMA data while being compatible with all null direct DM searches.

The $99_{S} \%$ CL compatibility of DAMA with the null searches is determined by the significant enhancement of the coupling to protons with respect to the coupling to neutrons, occurring for natural choices of the pseudo-scalar coupling to quarks. It is intriguing to notice that our results could also be extended to the case of massless mediator since the typical momentum transfer in direct detection is of the order of $m_{a}$.

Since the phenomenological success of this model relies on the enhancement of the DM-proton coupling respect to the DM-neutron one, as well as on the adopted nuclear form factor, a careful assessment of uncertainties and corrections to these quantities is in order.

The model could be tested with measurements of rare meson decays, flavor changing processes, and searches for axion-like particles with mass in the $\mathrm{MeV}$ range. In particular, Ref. ${ }^{24}$ investigates in detail the constraints arising from rare meson decays and fixed target experiments for different coupling structures between the pseudoscalar and quarks. They found that the entire parameter space in the right panel of Fig. 1 is excluded. Nevertheless, since the favored values of the pseudo-scalar mass in Fig. 1 are of the same order as the typical momentum transfer in direct searches experiments, the NR Sommerfeld enhancement can play a central role in re-opening the parameter space. This needs further studies in order to figure out whether the tantalizing possibility to reconcile DAMA results with the constraints coming from LUX and XENON100 discussed in this work, is still a vialable mechanism.

\section{References}

1. D. S. Akerib et al. [LUX Collaboration], [arXiv:1310.8214 [astro-ph.CO]].

2. E. Aprile et al. [XENON100 Collaboration], Phys. Rev. Lett. 109 (2012) 181301, [arXiv:1207.5988].

3. R. Agnese et al. [SuperCDMS Collaboration], Phys. Rev. Lett. 112241302 (2014), [arXiv:1402.7137 [hep-ex]].

4. S. Archambault et al. [PICASSO Collaboration], Phys. Lett. B 711153 (2012), [arXiv:1202.1240 [hep-ex]].

5. M. Felizardo et al., Phys. Rev. Lett. 108201302 (2012), [arXiv:1106.3014 [astroph.CO]].

6. E. Behnke et al. [COUPP Coll.], Phys. Rev. D 86 (2012) 052001, [arXiv:1204.3094].

7. S. C. Kim et al., Phys. Rev. Lett. 108181301 (2012), [arXiv:1204.2646 [astro-ph.CO]].

8. C. E. Aalseth et al. [CoGeNT Collaboration], [arXiv:1401.3295 [astro-ph.CO]].

9. C. E. Aalseth et al. [CoGeNT Collaboration], [arXiv:1401.6234 [astro-ph.CO]].

10. G. Angloher et al. [CRESST Collaboration], [arXiv:1109.0702 [astro-ph.CO]].

11. R. Agnese et al. [CDMS Coll.], [arXiv:1304.4279 [hep-ex]]. 
12. R. Bernabei et al., Eur. Phys. J. C 732648 (2013), [arXiv:1308.5109 [astro-ph.GA]].

13. A. K. Drukier, K. Freese and D. N. Spergel, Phys. Rev. D 333495 (1986).

14. A. L. Fitzpatrick, W. Haxton, E. Katz, N. Lubbers and Y. Xu, JCAP 1302 (2013) 004, [arXiv:1203.3542 [hep-ph]].

15. A. L. Fitzpatrick, W. Haxton, E. Katz, N. Lubbers, Y. Xu, [arXiv:1211.2818 [hep-ph]].

16. M. Cirelli, E. Del Nobile and P. Panci, JCAP 1310019 (2013), [arXiv:1307.5955 [hep-ph]].

17. P. Panci, Adv. High Energy Phys. 2014681312 (2014), [arXiv:1402.1507 [hep-ph]].

18. R. Catena and P. Gondolo, JCAP 1409045 (2014), [arXiv:1405.2637 [hep-ph]].

19. M. I. Gresham and K. M. Zurek, Phys. Rev. D 89123521 (2014), [arXiv:1401.3739 [hep-ph]].

20. H. Y. Cheng and C. W. Chiang, JHEP 1207009 (2012), [arXiv:1202.1292 [hep-ph]].

21. C. Boehm, M. J. Dolan, C. McCabe, M. Spannowsky and C. J. Wallace, JCAP 1405 009 (2014), [arXiv:1401.6458 [hep-ph]].

22. S. Chang, A. Pierce and N. Weiner, JCAP 1001006 (2010), [arXiv:0908.3192 [hep$\mathrm{ph}]]$.

23. C. Arina, E. Del Nobile and P. Panci, Phys. Rev. Lett. 114011301 (2015), [arXiv:1406.5542 [hep-ph]].

24. M. J. Dolan, F. Kahlhoefer, C. McCabe and K. Schmidt-Hoberg, JHEP 1503, 171 (2015), [JHEP 1507, 103 (2015)], [arXiv:1412.5174 [hep-ph]]. 